\title{
EXISTENCE THEOREMS FOR WEAK AND USUAL OPTIMAL SOLUTIONS IN LAGRANGE PROBLEMS WITH UNILATERAL CONSTRAINTS. II. EXISTENCE THEOREMS FOR WEAK SOLUTIONS $\left({ }^{1}\right)$
}

\author{
BY
LAMBERTO CESARI
}

Introduction. In the present paper II we prove existence theorems for weak optimal solutions of nonparametric Lagrange problems with (or without) unilateral constraints.

In paper $\mathrm{I}([\mathrm{e}]$ of the reference list) we considered arbitrary pairs $x(t), u(t)$ of vector functions, $u(t)$ measurable with values in $E_{m}, x(t)$ absolutely continuous with values in $E_{n}$, and we discussed the existence of the absolute minimum of the functional

$$
I[x, u]=\int_{t_{1}}^{t_{2}} f_{0}(t, x(t), u(t)) d t,
$$

with side conditions represented by a differential system

$$
d x / d t=f(t, x(t), u(t)), \quad t_{1} \leqq t \leqq t_{2},
$$

with constraints

$$
(t, x(t)) \in A, \quad u(t) \in U(t, x(t)), \quad t_{1} \leqq t \leqq t_{2},
$$

and boundary conditions

$$
\left(t_{1}, x\left(t_{1}\right), t_{2}, x\left(t_{2}\right)\right) \in B,
$$

where $A$ is a given closed subset of the $t x$-space $E_{1} \times E_{n}$, where $B$ is a given closed subset of the $t_{1} x_{1} t_{2} x_{2}$-space $E_{2 n+2}$, and where $U(t, x)$ denotes a given closed variable subset of the $u$-space $E_{m}$, depending on time $t$ and space $x$. The set $A$ may coincide with the whole space $E_{1} \times E_{m}$, and $U$ may be fixed and coincide with the whole space $E_{m}$.

In the present paper II we discuss the same problem for weak (or generalized) solutions, introduced by R. V. Gamkrelidze [3] as measurable probability distributions of usual solutions (chattering states), and by L. C. Young [14], E. J. McShane [5], J. Warga [12], and T. Wazewski [13] by analogous processes. In the particular situation where $U(t, x)$ is compact for every $(t, x)$, we obtain

Received by the editors December 6, 1965.

(1) Research partially supported by NSF-grant GP-3920 at The University of Michigan. 
weak solutions for Pontryagin's problems; in the particular situation where $U$ is fixed and coincides with the whole space, we obtain weak solutions for problems which have essentially the same generality as usual Lagrange problems. Throughout this paper we shall assume $U(t, x)$ to be any closed subset of $E_{m}$.

In the present paper II we first discuss the question as to whether the infimum of the (generalized) functional for generalized solutions is the same as the infimum of the functional for usual solutions. Since here the control space $U(t, x)$ depends on both $t$ and $x$, and $U$ is a closed (not necessarily compact) set, the question is particularly difficult. A sufficient condition for equality is given. We then prove existence theorems for the existence of generalized optimal solutions.

To simplify references we continue the numeration of sections of the previous paper I. In referring to this paper we shall use I followed by section number.

In subsequent papers we shall extend some of the present results to multidimensional Lagrange problems involving partial differential equations in Sobolev's spaces with unilateral constraints.

14. Weak solutions. Instead of considering the usual cost functional, differential equations, and constraints

$$
\begin{gathered}
I[x, u]=\int_{t_{1}}^{t_{2}} f_{0}(t, x(t), u(t)) d t, \\
d x / d t=f(t, x(t), u(t)), \quad f=\left(f_{1}, \cdots, f_{n}\right), \\
(t, x(t)) \in A, \quad u(t) \in U(t, x(t)),
\end{gathered}
$$

we shall consider a new cost functional, differential equations, and constraints

$$
\begin{gathered}
J(x, p, v)=\int_{t_{1}}^{t_{2}} g_{0}(t, x(t), p(t), v(t)) d t, \\
d x / d t=g(t, x(t), p(t), v(t)), \quad g=\left(g_{1}, \cdots, g_{n}\right), \\
(t, x(t)) \in A, \quad v(t) \in V(t, x(t)), \quad p(t) \in \Gamma,
\end{gathered}
$$

where

$$
\begin{aligned}
g_{0}(t, x, p, v) & =\sum_{j=1}^{v} p_{j} f_{0}\left(t, x, u^{(j)}\right), \\
g(t, x, p, v) & =\sum_{j=1}^{v} p_{j} f\left(t, x, u^{(j)}\right) .
\end{aligned}
$$

Precisely, $v(t)=\left(u^{(1)}, \cdots, u^{(v)}\right)$ represents a finite system of some $v \geqq n+1$ functions $u^{(1)}(t), \cdots, u^{(v)}(t)$, each $u^{(j)}$ having its values in $U$, that is, $u^{(j)}(t) \in U(t, x(t))$ $\subset E_{m}, j=1, \cdots, v$. Thus, we think of $v=\left(u^{(1)}, \cdots, u^{(v)}\right)$ as a vector variable whose $v$ components $u^{(1)}, \cdots, u^{(v)}$ are themselves vectors $u$ with values in $U(t, x)$. In other words, 


$$
\begin{gathered}
v=\left(u^{(1)}, \cdots, u^{(v)}\right), \quad u^{(j)} \in U(t, x), \quad j=1, \cdots, v, \text { or } \\
v \in V(t, x)=[U(t, x)]^{v}=U \times \cdots \times U \subset E_{m v},
\end{gathered}
$$

where $U^{v}$ is the product space of $U$ by itself $v$-times, and thus $V$ is a subset of the Euclidean space $E_{m v}$. In (2) $p=\left(p_{1}, \cdots, p_{v}\right)$ represents a probability distribution. Hence, $p=\left(p_{1}, \cdots, p_{v}\right)$ is a point of the simplex $\Gamma$ of the Euclidean space $E_{v}$ defined by $p_{j} \geqq 0, p_{1}+\cdots+p_{v}=1$. Finally, in (2), the new control variable is $(p, v)$ with values $(p, v) \in \Gamma \times V(t, x) \subset E_{v+m v}$. In (2) $g=\left(g_{1}, \cdots, g_{n}\right)$, and all $g_{0}, g_{1}, \cdots, g_{n}$ are defined by

$$
g_{i}(t, x, p, v)=\sum_{j=1}^{v} p_{j} f_{i}\left(t, x, u^{(j)}\right), \quad i=0,1, \cdots, n .
$$

As usual we shall require that the functions $p(t), v(t), t_{1} \leqq t \leqq t_{2}$, are measurable, that $x(t), t_{1} \leqq t \leqq t_{2}$, is absolutely continuous, that the differential system (2) is satisfied almost everywhere (a.e.), and that $g_{0}(t, x(t), p(t), v(t))$ is $L$-integrable together with all $g_{i}, i=1, \cdots, n$. When needed, we shall require as usual that $x(t)$ satisfies boundary conditions of the type $\left(t_{1}, x\left(t_{1}\right), t_{2}, x\left(t_{2}\right)\right) \in B \subset E_{2 n+2}$ where $B$ is a given closed subset of $E_{2 n+2}$.

We say that $[p(t), v(t)]$ is a generalized strategy, that $p(t)$ is a probability distribution, and that $x(t)$ is a generalized trajectory. We shall also say, for the sake of brevity, that $[x(t), p(t), v(t)]$ is a weak solution.

If we introduce, as usual, the auxiliary variable $x^{0}$ with initial value $x^{0}\left(t_{1}\right)=0$ and the vector $\tilde{x}=\left(x^{0}, x\right)=\left(x^{0}, x^{1}, \cdots, x^{n}\right)$, then instead of the usual system $d \tilde{x} / d t=\tilde{f}$ we shall consider the system

$$
d \tilde{x} / d t=\tilde{g}(t, x(t), p(t), v(t)), \quad \tilde{g}=\left(g_{0}, g\right)=\left(g_{0}, g_{1}, \cdots, g_{n}\right),
$$

and we have $J[x, p, v]=x^{0}\left(t_{2}\right)$. Instead of the usual sets $Q(t, x)=$ $f[t, x, U(t, x)] \subset E_{n}$, and

$$
\widetilde{Q}(t, x)=\tilde{f}[t, x, U(t, x)]=\left[\tilde{z}=\left(z^{0}, z\right) \mid \tilde{z}=\tilde{f}(t, x, u), u \in U(t, x)\right] \subset E_{n+1},
$$

we shall now consider the sets

$$
\begin{gathered}
R(t, x)=g[t, x, \Gamma \times V(t, x)]=[z \mid z=g(t, x, p, v),(p, v) \in \Gamma \times V(t, x)] \subset E_{n}, \\
\tilde{R}(t, x)=\tilde{g}[t, x, \Gamma \times V(t, x)]=\left[\tilde{z}=\left(z^{0}, z\right) \mid \tilde{z}=\tilde{g}(t, x, p, v),(p, v) \in \Gamma \times V(t, x)\right] \subset E_{n+1} .
\end{gathered}
$$

Since

$\tilde{R}(t, x)=\left[\tilde{z}=\left(z^{0}, z\right) \mid \tilde{z}=\sum_{j=1}^{v} p_{j} \tilde{f}\left(t, x, u^{(j)}\right), p \in \Gamma, u^{(j)} \in U(t, x), j=1, \cdots, v\right]$

with $v \geqq n+1$, we see that $\widetilde{R}(t, x)$ is the convex hull of the set $\widetilde{Q}(t, x)$ in $E_{n+1}$, and hence $\widetilde{R}(t, x)$ is always convex. For weak solutions there is no reason, therefore, to consider sets analogous to the sets $\widetilde{Q}(t, x)$.

It is important to show that any (ordinary) strategy $u(t)$ and corresponding 
(ordinary) trajectory $x(t)$ (thus, satisfying (1)) can be interpreted as a generalized trajectory, and this can be done by taking $v(t)=\left(u^{(j)}(t), j=1, \cdots, v\right)$ defined by $u^{(j)}(t)=u(t), p^{(j)}(t)=1 / v, j=1, \cdots, v$. Then relations (2) reduce to relations (1), the trajectory is still $x(t)$ and $J[x, p, v]=I[x, u]$.

Instead of the usual set $M$ we shall now consider the set $N \subset E_{1+n+v+m v}$ of all $(t, x, p, v)$ with $(t, x) \in A, p \in \Gamma, v \in V(t, x)$. As usual, we shall assume that $A$ is a closed subset of $E_{1} \times E_{n}$, and that $\tilde{f}=\left(f_{0}, f_{1}, \cdots, f_{n}\right)$ is a continuous vector function from $M$ into $E_{n+1}$.

Let $\Omega$ be the class of all admissible pairs $\left[\tilde{x}(t)=\left(x^{0}, x\right), u(t)\right]$, and let $\Omega^{*}$ be the class of all generalized systems $\left[\tilde{y}(t)=\left(y^{0}, y\right), p(t), v(t)\right]$ satisfying corresponding differential equations, constraints, and boundary conditions. As mentioned above, we have $\Omega \subset \Omega^{*}$. If

$$
i=\underset{\Omega}{\operatorname{Inf}} I[x, u], \quad j=\operatorname{Inf}_{\mathbf{\Omega}^{*}} J[x, p, v],
$$

then $\Omega \subset \Omega^{*}$ implies $i \geqq j$.

It is a fairly general phenomenon that generalized trajectories $y(t)$ and corresponding values of $J[y, p, v]=y^{0}\left(t_{2}\right)$ of the cost functional $J$ can be approached by means of usual trajectories $x(t)$ and corresponding values of the cost functional $I[x, u]$, so that $i=j$.

Property $(P)$. We shall say that property $(P)$ holds provided $j=i$.

(i) (R. V. Gamkrelidze [3]). Under the hypotheses that $A=E_{1} \times E_{n}$, that $U(t, x)$ depends on $t$ only, that $U(t)$ is compact for every $t$, that $U(t)$ is an upper semicontinuous function of $t$, that $f$ satisfies a Lipschitz condition, and that $\Omega$ is the class of all admissible pairs $x(t), u(t)$, then property $(P)$ holds.

In the present more general situation $-A$ any closed set, $U(t, x)$ depending both on $t$ and $x, U(t, x)$ closed, $U(t, x)$ satisfying condition $(U)$ - property $(P)$ is more difficult to prove, even under additional hypotheses. Actually, property $(P)$ is not valid in general as the following simple example shows.

Take $m=1, n=2, U$ made up of only two points $u=-1$ and $u=1$, $A=E_{1} \times A_{0}$, where $E_{1}$ is the $t$-axis, and $A_{0}$ is made up of the three sides of the triangle of vertices $(0,0),(1,1),(2,0)$ of the $x y$-plane $E_{2}, I=\int_{t_{1}}^{t_{2}} y|u| d t$, differential system $d x / d t=1, d y / d t=u$, and boundary conditions $t_{1}=0, x(0)$ $=y(0)=0, x\left(t_{2}\right)=2, y\left(t_{2}\right)=0$. Then $\Omega$ is made up of only one element: $[x(t), y(t), u(t), \quad 0 \leqq t \leqq 2] \quad$ with $\quad x(t)=t, \quad y(t)=t, \quad u(t)=1 \quad$ if $\quad 0 \leqq t \leqq 1$, $x(t)=t, y(t)=2-t, u(t)=-1$ if $1<t \leqq 2$, and for this only element $I=1$. On the other hand, $\Omega^{*}$ contains other elements, in particular, $\left[p_{1}(t)=p_{2}(t)=1 / 2\right.$, $\left.u^{(1)}(t)=1, u^{(2)}(t)=-1, x(t)=t, y(t)=0,0 \leqq t \leqq 2\right]$ for which $J=0$. Thus $i=1$ and $j=0$. Another example of an analogous situation has been given by A. Plis, Trajectories and quasitrajectories of an orientor field, Bull. Acad. Polon. Sci. 11 (1963), 369-370.

Nevertheless, we shall prove property $(P)$ under a set of requirements which 
are all satisfied in most cases and which are easier to verify than property $(P)$. Our statement (iv of $\$ 15$ below) contains statement (i) of R. V. Gamkrelidze [3] as a particular case.

15. Sufficient conditions for property $(P)$. We shall start with a few remarks on properties $(U)$ and $(Q)$ for the sets $\tilde{R}(t, x)$.

(i) If $A$ is a compact subset of $E_{1} \times E_{n}$, if $U(t, x)$ is a compact subset of $E_{m}$ for every $(t, x)$ in $A$, and $U(t, x)$ is an upper semicontinuous function of $(t, x)$ in $A$, then (a) $V(t, x)$ is also a compact subset of $E_{m v}$ for every $(t, x) \in A$; (b) $V(t, x)$ is an upper semicontinuous function of $(t, x)$ in $A$; (c) $N$ is compact; (d) $R(t, x)$ is a compact convex subset of $E_{n}$ and $\tilde{R}(t, x)$ is a compact convex set of $E_{n+1}$; (e) $R(t, x)$ and $\tilde{R}(t, x)$ are upper semicontinuous functions of $(t, x)$ in $A$.

Proof. The part concerning $V$ is a corollary of (I, $\S 4$, (viii)). The part concerning $N$ is a corollary of $(\mathrm{I}, \S 4$, (vii)). For the part concerning $R$ and $\widetilde{R}$ we have only to observe that $R(t, x)[\widetilde{R}(t, x)]$ is the set of all $z=\left(z^{1}, \cdots, z^{n}\right)\left[\tilde{z}=\left(z^{0}, z^{1}, \cdots, z^{n}\right)\right]$ with

$$
z=\sum_{j} p_{j} f\left(t, x, u^{(j)}\right)\left[\tilde{z}=\sum_{i} p_{j} \tilde{f}\left(t, x, u^{(j)}\right)\right],
$$

where $\Sigma_{j}$ ranges over all $j=1, \cdots, v$, where $p_{j} \geqq 0, p_{1}+\cdots+p_{v}=1$, the $v$ points $f\left(t, x, u^{(j)}\right)$ are all in $Q(t, x)[\widetilde{Q}(t, x)]$, and $v \geqq n+1$. Thus, $R[\tilde{R}]$ is the convex hull of $Q[\tilde{Q}]$. Since $Q$ is compact, also $R[\tilde{R}]$ is compact. Since $Q$ and $\widetilde{Q}$ are known to be upper semicontinuous functions of $(t, x)$, we deduce from (I, $\S 4$, (viii)) that $R$ and $\widetilde{R}$ have the same property.

(ii) If $A$ is a closed subset of $E_{1} \times E_{n}$, if $U(t, x)$ is a closed subset of $E_{m}$ for every $(t, x) \in A$, and $U(t, x)$ satisfies condition $(U)$ in $A$, then (a) $V(t, x)$ is also a closed subset of $E_{m v}$ for every $(t, x) \in A$; (b) $V(t, x)$ satisfies property ( $U$ ) in $A$; (c) $N$ is closed; (d) $R(t, x)$ is a convex subset of $E_{n}$ and $\widetilde{R}(t, x)$ is a convex subset of $E_{n+1}$.

Proof. The part concerning $V$ is a corollary of (I, $\$ 4$, (iv)). The part concerning $N$ is a corollary of (I, $\S 4$, (ii)). For the part concerning $R$ and $\widetilde{R}$ we have only to repeat the convexity argument of the previous proof.

REMARK. Under the conditions of (ii) the set $R(t, x)$ does not necessarily satisfy property $(Q)$, actually $R$ and $\widetilde{R}$ may not even be closed as the following example shows. This example is the analogous of one of $(I, \S 4,(C))$. Take $m=n=1, A=[-1 \leqq t \leqq 1,0 \leqq x \leqq 1]$, let $U(t, x)$ be the fixed interval $U$ $=\left[u \in E_{1} \mid 0 \leqq u<+\infty\right]$ and $f=(u+1)^{-1}-t$. Then $Q(t, x)=\left[z \in E_{1} \mid-t\right.$ $<z \leqq 1-t]$ is a convex set, and $R(t, x)=Q(t, x)$. Here $Q(t, x)$ is not closed, and hence $R$ and $Q$ satisfy neither property $(U)$ nor property $(Q)$. If we take $f_{0}=0$, then the corresponding sets $\widetilde{R}=\widetilde{Q}$ again are not closed nor satisfy properties $(U)$ or $(Q)$.

Even the stronger hypothesis that $A$ is compact, that $f$ is continuous on $M$, that $U(t, x)$ has property $(Q)$ in $A$, and $Q(t, x)$ is compact and convex for every 
$(t, x) \in A$ does no mply that $Q(t, x)$ has property $(Q)$ in $A$ as we have seen in I, $\S 4,(\mathrm{C})$. Since again $R=Q$, we see that the same hypothesis does not imply that $R$, or $\widetilde{R}$, have property $(Q)$.

We shall now prove property $(P)$ (statement (iv)) below. We need first to list a number of requirements which are usually satisfied, and are fairly easy to verify in any particular situation.

Property $\left(p_{1}\right)$. For every generalized element $\left[\tilde{x}(t)=\left(x^{0}, x\right), p(t), v(t)\right.$, $\left.t_{1} \leqq t \leqq t_{2}\right] \in \Omega^{*}$ and $\varepsilon>0$ there is another generalized element $\left[\tilde{y}(t)=\left(y^{0}, y\right)\right.$, $\left.q(t), w(t), t_{1}^{\prime} \leqq t \leqq t_{2}^{\prime}\right]$ and a constant $M>0$ (both depending on $\varepsilon$ and the given element), such that the graph $\left[(t, y(t)), t_{1}^{\prime} \leqq t \leqq t_{2}^{\prime}\right]$ lies in the interior of $A$, (or in the interior of $A$ relatively to the slab $\left.t_{1} \leqq t \leqq t_{2}, x \in E_{n}\right),|w(t)| \leqq M$, and $\tilde{y}(t), t_{1}^{\prime} \leqq t \leqq t_{2}^{\prime}$, lies in the closed $\varepsilon$-neighborhood of $\tilde{x}(t), t_{1} \leqq t \leqq t_{2}$, in the $\rho$-metric, that is, $\rho(\tilde{y}, \tilde{x}) \leqq \varepsilon$. In particular $|J(\tilde{y}, q, w)-J(\tilde{x}, p, v)| \leqq \varepsilon$.

We shall use this property in the proof of property $(P)$. Indeed, after having approached any given generalized element $(\tilde{x}, p, v)$ by another generalized element $(\tilde{y}, q, w)$ as above we shall replace the latter by an usual admissible pair $\left[z(t), u(t), t_{1}^{\prime} \leqq t \leqq t_{2}^{\prime}\right]$ with $|\tilde{z}(t)-\tilde{y}(t)| \leqq \eta$ for all $t_{1}^{\prime} \leqq t \leqq t_{2}^{\prime}$. It is now evident that $x(t)$ will not satisfy exactly the boundary conditions since we know only that the end points $\left[t_{1}^{\prime}, z\left(t_{1}^{\prime}\right)\right]$ and $\left[t_{2}^{\prime}, z\left(t_{2}^{\prime}\right)\right]$ of $z$ are at a distance $\leqq \varepsilon+\eta$ from the endpoints $\left[t_{1}, x\left(t_{1}\right)\right],\left[t_{2}, x\left(t_{2}\right)\right]$ of $x$, these satisfying the boundary conditions exactly since $x$ belongs to $\Omega^{*}$. We have to guarantee that this relaxation in the boundary conditions will not essentially reduce the infimum $i$ of $I[x, u]$ in $\Omega$. Usually, this is the case.

Precisely, for any $\delta>0$ let us denote by $\Omega_{\delta}$ the class of all admissible pairs $\left[x(t), u(t), t_{1} \leqq t \leqq t_{2}\right]$ whose endpoints $\left[t_{1}, x\left(t_{1}\right)\right],\left[t_{2}, x\left(t_{2}\right)\right]$ are at a distance $\leqq \delta$ from the endpoints $\left[t_{1}^{\prime}, y\left(t_{1}^{\prime}\right)\right],\left[t_{2}, y\left(t_{2}^{\prime}\right)\right]$ of some generalized system $\left[\tilde{y}(t)=\left(y^{0}, y\right), p(t), v(t), t_{1}^{\prime} \leqq t \leqq t_{2}^{\prime}\right]$ of $\Omega^{*}$. Then $\Omega \subset \Omega_{\delta}$. Thus, we define

$$
i^{\prime}=\lim _{\delta \rightarrow 0+\Omega_{\delta}} \operatorname{Inf} I[x, u],
$$

and obviously $i^{\prime}$ exists and $i \geqq i^{\prime}$. Usually we have $i=i^{\prime}$. Besides, in particular situations, the class $\Omega_{\delta}$ can be restricted in various ways, so as to make this equality easier to verify.

Property $\left(p_{2}\right)$. For the given problem we have $i=i^{\prime}$.

This is a much easier property to verify than property $(P)$. For instance, for problems with $t_{1}, t_{2}, x\left(t_{1}\right)=x_{10}, x\left(t_{2}\right)=x_{20}$ all fixed, and $A=\left[(t, x) \mid t_{1} \leqq t \leqq t_{2}\right.$, $\left.\left|x-x_{20}\right| \leqq\left|t-t_{2}\right|\right]$, with $\left|x_{10}-x_{20}\right|<\left|t_{1}-t_{2}\right|$, we may require in $\left(p_{1}\right)$ that $t_{1}^{\prime}=t_{1}, y\left(t_{1}\right)=x\left(t_{1}\right)=x_{10}$, and then $\left(p_{2}\right)$ is certainly satisfied if the point $x_{20} \in E_{n}$ has the following property: $(q)$. Given $\varepsilon>0$ there is a $\delta>0$ such that for any point $(\bar{t}, \bar{x}) \in A$ at a distance $\leqq \delta$ from $\left(t_{2}, x_{20}\right)$, there is a trajectory $x(t), \bar{t} \leqq t \leqq t_{2}$, joining $\bar{x}$ to $x_{20}$ [that is, admissible pair $x(t), u(t), \bar{t} \leqq t \leqq t_{2}$, with $\left.x(\bar{t})=\bar{x}, x\left(t_{2}\right)=x_{20}\right]$, with $|I[x, u]|<\varepsilon$. This property $(q)$ is a "local property of controllability in the small" which is easy to verify in most cases. 
We sha!l now need a local Lipschitz condition of $f(t, x, u)$. Precisely we shall require:

Property $\left(p_{3}\right)$. Given $N>0$, there is another constant $L \geqq 0$ such that $|\tilde{f}(t, x, u)-\tilde{f}(t, y, v)| \in L(|x-y|+|u-v|) \quad$ for all $(t, x, u),(t, y, v) \in M$ with $|x|,|y| \leqq N,|u|,|v| \leqq N$. If $U$ depends on $t$ only, or $U=U(t)$, then we require only

$$
|f(t, x, u)-f(t, y, u)| \leqq L|x-y|
$$

for all $(t, x, u),(t, y, u) \in M$, with

$$
|x|,|y| \leqq N,|u| \leqq N
$$

We shall finally require a property of continuity for the set $U(t, x)$ as a function of $x$. The condition we require is trivially satisfied when $U(t, x)$ depends on $t$ only.

Property $\left(p_{4}\right)$. Given $N>0$ there is another constant $H \geqq 0$ such that for any two points $(t, x) \in A,(t, y) \in A,-N \leqq t \leqq N,|x|,|y| \leqq N$, and for any $u \in U(t, x)$ with $|u| \leqq N$, there is at least another point $v \in U(t, y)$ with $|u-v|$ $\leqq H|x-y|$.

(iii) Let $A$ be any closed subset of $E_{1} \times E_{n}$, let $U(t, x)$ be any closed subset of $E_{m}$ for every $(t, x) \in A$, satisfying condition $(U)$ in $A$, and let $\tilde{f}(t, x, u)=\left(f_{0}, \cdots, f_{n}\right)$ be any continuous vector function on $M$. Assume that properties $\left(p_{3}\right),\left(p_{4}\right)$ hold. Then for every element $\left[\tilde{y}(t)=\left(y^{0}, y\right), p(t), v(t), t_{1} \leqq t \leqq t_{2}\right]$ of $\Omega^{*}$ with $v(t)$ bounded and the graph $(t, y(t))$ lying in the interior of $A$, there is an admissible pair $\left[\tilde{x}(t)=\left(x^{0}, x\right), u(t), t_{1} \leqq t \leqq t_{2}\right]$ with $|\tilde{x}(t)-\tilde{y}(t)| \leqq \varepsilon$ for all $t_{1} \leqq t \leqq t_{2}$, in particular $|J-I|=\left|y^{0}\left(t_{2}\right)-x^{0}\left(t_{2}\right)\right| \leqq \varepsilon$. If $U(t, x)$ depends on $t$ only, then property $\left(p_{4}\right)$ is trivial.

Proof. We prove statement (iii) for the vector function $x(t)$. By a change of notations, this is the general case. Also, it is not restrictive to assume $t_{1}=0$, $t_{2}=b>0$. Let $x_{0}$ denote the initial value $x_{0}=y(0)$. The proof is divided into the parts (a) and (b).

(a) Let $2 d>0$ be the distance of the graph $G=[(t, y(t)), 0 \leqq t \leqq b]$ from the boundary of $A$ ( $d=1$ if $A$ is the whole $t x$-space). Let $A_{0}$ be the closed $d$-neighborhood of $G$. Then $G$ and $A_{0}$ are compact, $G$ lies in the interior of $A_{0}$, and $A_{0} \subset A$.

Since $v(t)=\left(u^{(j)}(t), j=1, \cdots, v\right)$ is bounded, there is a constant $N>0$ such that $\left|u^{(j)}(t)\right| \leqq N, 0 \leqq t \leqq b, j=1, \cdots, v$. Let $M_{0}$ be the set of all $(t, x, u)$ with $(t, x) \in A_{0}, \quad 0 \leqq t \leqq b, u \in U(t, x),|u| \leqq N$. Obviously $M_{0} \subset M$, and $M_{0}$ is a compact subset of $E_{1+n+m}$ (I, $\S 4$,ii). Also $M_{0}$ is certainly contained in the set of all $(t, x, u) \in M$ with $-N_{0} \leqq t \leqq N_{0},|x| \leqq N_{0}$ for some constant $N_{0}$.

By hypothesis $f(t, x, u)$ is a continuous function on $M_{0}$, hence bounded, say $|f(t, x, u)| \leqq N_{1}$, and also there is a constant $L \geqq 0$ such that $|f(t, x, u)-f(t, y, u)|$ $\leqq L|x-y|$ for all $(t, x, u),(t, y, u) \in M_{0}$.

We shall denote by $M_{1}$ the set of all $(t, x, u)$ with $(t, x) \in A_{0}, 0 \leqq t \leqq b, u \in U(t, x)$, $|u| \leqq N+1$.

Let $H$ be the number relative to property $\left(p_{4}\right)$ and $N_{0}$. Given $\varepsilon>0$ let $\delta>0$ be 
any number such that $\delta e^{L(H+1) b} \leqq \min [d, \varepsilon], \delta H e^{L(H+1) b} \leqq 1$.

Let $\varepsilon_{1}=\min [d, \varepsilon, \delta]$ and take $\sigma>0$ so that $3 \sigma \leqq \varepsilon_{1}$ and $\varepsilon \sigma e^{L b} \leqq \varepsilon_{1}$.

Let $\eta_{1}=(2 b)^{-1} \sigma$, and let $\delta_{1}>0$ be a number such that

$$
\left|f(t, x, u)-f\left(t^{\prime}, x^{\prime}, u^{\prime}\right)\right| \leqq \eta_{1}
$$

for all $(t, x, u),\left(t^{\prime}, x^{\prime}, u^{\prime}\right) \in M_{1}$ at a distance $\leqq \delta_{1}$.

Let $\eta_{2}=(2 N)^{-1} \sigma$. Since the $v$ functions $u^{(j)}(t)$ are measurable in $[0, b]$, there is a closed subset $K$ of $[0, b]$ with meas $K>b-\eta_{2}$, such that the $v$ functions $u^{(j)}(t), j=1, \cdots, v$, are continuous on $K$. Then, $K$ is compact, and the $v$ functions $u^{(j)}(t), j=1, \cdots, v$, are uniformly continuous on $K$. Then there is some $\delta_{2}>0$ such that $\left|u^{(j)}(t)-u^{(j)}\left(t^{\prime}\right)\right| \leqq \delta_{1}$ for all $j=1, \cdots, v$, and all $t, t^{\prime} \in K$ with $\left|t-t^{\prime}\right| \leqq \delta_{2}$. Also, we can take $\delta_{2} \leqq \delta_{1}$ so small that $t, t^{\prime} \in[0, b],\left|t-t^{\prime}\right| \leqq \delta_{2}$, implies $\left|y(t)-y\left(t^{\prime}\right)\right| \leqq \delta_{1}$.

Let us divide $I=[0, b]$ into $k$ equal consecutive intervals, say $I_{k s}, s=1, \cdots, k$, each of length $b / k$. For each $I_{k s}$, let $\left[\varepsilon_{k s j}, j=1, \cdots, v\right]$ be any subdivision of $I_{k s}$ into $v$ measurable disjoint subsets $\varepsilon_{k s j} \subset I_{k s}$ (for instance, subintervals), such that

$$
\text { meas } \varepsilon_{k s j}=\int_{I_{k_{e}}} p_{j}(t) d t, \quad j=1, \cdots, v
$$

Then

$$
\sum_{j} \operatorname{meas} \varepsilon_{k s j}=\sum_{j} \int_{I_{k_{-}}} p_{j}(t) d t=\int_{I_{k_{-}}} d t=\operatorname{meas} I_{k s} .
$$

We take now

$$
u(t)=u^{(j)}(t) \text { for all } t \in \varepsilon_{k s j}, \quad j=1, \cdots, v, s=1, \cdots, k .
$$

Then $u(t), t_{1} \leqq t \leqq t_{2}$, is a measurable function in $I$ with values $u(t) \in U(t, y(t))$, $t_{1} \leqq t \leqq t_{2}$, and $|u(t)| \leqq N$. Let us consider the differential system

$$
d x / d t=f(t, y(t), u(t)), \quad 0 \leqq t \leqq b,
$$

with initial value $x(0)=y(0)=x_{0}$. Since $\left(0, x_{0}\right)$ is an interior point of $A_{0}$, the solution $x(t)$ of (4) exists in a right neighborhood of $t=0$, say $[0, \bar{t}]$, and $(t, x(t))$ lies in $A_{0}$.

Let $k_{2}$ be the smallest integer such that $b / k_{2} \leqq \delta_{2}$. Hence, for $k \geqq k_{2}$ and for any two points $t, t^{\prime} \in I_{k s 0}=I_{k s} \cap K$, we have $\left|t-t^{\prime}\right| \leqq b / k \leqq \delta_{2}$, and

$$
\left|u^{(j)}(t)-u^{(j)}\left(t^{\prime}\right)\right| \leqq \delta_{1}, \quad j=1, \cdots, v .
$$

Let $I_{k s 0}=I_{k s} \cap K, \varepsilon_{k s j 0}=\varepsilon_{k s j} \cap K, I_{k s}^{\prime}=I_{k s j}-K, \varepsilon_{k s j}^{\prime}=\varepsilon_{k s j}-K, K^{\prime}=I-K$. For any triple $k, s, j$ with meas $\varepsilon_{k s j 0}>0$ we select a point $\tau=\tau_{k s j} \in \varepsilon_{k s j 0}$. If meas $\varepsilon_{k s j 0}=0$, we do not select any point, and actually we disregard in the lines below the corresponding term. Obviously, meas $K^{\prime} \leqq \eta_{2}$.

For any interval $[0, t]$ let $K_{t 0}, K_{t}^{\prime}$ be the sets $\left.K_{t 0}=K \cap[0, t)\right], K_{t}^{\prime}=[0, t]-K$.

Note that $\int_{I_{k s}} p_{j}(\xi) d \xi-$ meas $\varepsilon_{k s j}=0$, and hence 


$$
\begin{aligned}
& \underset{s}{\sum \sum_{j}} \mid \int_{I_{k s 0}} p_{j}(\xi) d \xi-\text { meas } \varepsilon_{k s j 0} \mid \\
& \quad=\sum_{s} \sum_{j}\left|\left(\int_{I_{k s}} p_{j}(\xi) d \xi-\operatorname{meas} \varepsilon_{k s j}\right)-\int_{I^{\prime} k_{s}} p_{j}(\xi) d \xi+\operatorname{meas} \varepsilon_{k s j}^{\prime}\right| \\
& \quad \leqq 0+\sum_{s} \int_{I^{\prime}{ }_{k s}} \sum_{j} p_{j}(\xi) d \xi+\underset{j}{\sum} \sum_{s} \operatorname{meas} \varepsilon_{k_{s j}}^{\prime} \leqq 2 \operatorname{meas} K^{\prime} \leqq 2 \eta_{2} .
\end{aligned}
$$

Now $x(t)$ and $y(t)$ are absolutely continuous with $x(0)=y(0)=x_{0}$, and

$$
\begin{aligned}
& d y / d t=g(t, y(t), p(t), v(t))=\sum_{j} p_{j}(t) f\left(t, y(t), u^{(j)}(t)\right), \quad 0 \leqq t \leqq b, \\
& d x / d t=f(t, y(t), u(t)), \quad 0 \leqq t<\bar{t}, \quad x(0)=y(0)=x_{0} .
\end{aligned}
$$

For any $t$ we have $u(t) \in U(t, y(t))$, hence $f(t, y(t), u(t))$ is defined, and

$$
\begin{aligned}
y(t)-x(t) & =\int_{0}^{t}[g(\xi, y(\xi), p(\xi), v(\xi))-f(\xi, y(\xi), u(\xi))] d \xi \\
& =\left(\int_{K_{t}}+\int_{K^{\prime} t}\right)\left[\sum_{j} p_{j}(\xi) f\left(\xi, y(\xi), u^{(j)}(\xi)\right)-f(\xi, y(\xi), u(\xi))\right] d \xi \\
& =\mu_{1}+\mu_{2} .
\end{aligned}
$$

Since $|f| \leqq N$ in $M_{0}$, we have

$$
\begin{aligned}
\left|\mu_{2}\right| & =\left|\int_{K^{\prime}}\left[\sum_{j} p(\xi) f\left(\xi, y(\xi), u^{(j)}(\xi)\right)-f(\xi, y(\xi), u(\xi))\right] d \xi\right| \\
& \leqq \int_{K^{\prime}}\left[\sum_{j} p_{j}(\xi)\left|f\left(\xi, y(\xi), u^{(j)}(\xi)\right)\right|+|f(\xi, y(\xi), u(\xi))|\right] d \xi \\
& \leqq 2 N \text { meas } K_{t}^{\prime} \leqq 2 N \eta_{2}=\sigma .
\end{aligned}
$$

Also, we have

$$
\begin{aligned}
\left|\mu_{1}\right|= & \left|\int_{K_{t}}\left[\sum_{j} p_{j}(\xi) f\left(\xi, y(\xi), u^{(j)}(\xi)\right)-f(\xi, y(\xi), u(\xi))\right] d \xi\right| \\
= & \left|\sum_{s} \sum_{j}\left\{\int_{I_{k s 0}} p_{j}(\xi) f\left(\xi, y(\xi), u^{(j)}(\xi)\right) d \xi-\int_{\varepsilon_{k s j}} f\left(\xi, y(\xi), u^{(j)}(\xi)\right) d \xi\right\}\right| \\
= & \mid \sum_{s} \sum_{j}\left\{f\left(\tau, y(\tau), u^{(j)}(\tau)\right)\left[\int_{I_{k s 0}} p_{j}(\xi) d \xi-\text { meas } \varepsilon_{k s j 0}\right]\right\} \\
& +\underset{s}{\Sigma} \sum_{j} \int_{I_{k s 0}} p_{j}(\xi)\left[f\left(\xi, y(\xi), u^{(j)}(\xi)\right)-f\left(\tau, y(\tau), u^{(j)}(\tau)\right)\right] d \xi \\
& +\underset{s}{\Sigma} \sum_{j} \int_{\varepsilon_{k s j o}}\left[f\left(\xi, y(\xi), u^{(j)}(\xi)\right)-f\left(\tau, y(\tau), u^{(j)}(\tau)\right)\right] d \xi \mid,
\end{aligned}
$$


where summations and integrations are extended only over those terms and intervals concerning $[0, t]$. In each bracket of the second and third sums of the last member we have $|\xi-\tau| \leqq b / k \leqq \delta_{2} \leqq \delta_{1}$, hence $|y(\xi)-y(\tau)| \leqq \delta_{1},\left|u^{(j)}(\xi)-u^{(j)}(\tau)\right| \leqq \delta_{1}$ and each bracket has absolute value $\leqq \eta_{1}$. By using this remark and (5) we obtain

Thus

$$
\begin{aligned}
\left|\mu_{1}\right| & \leqq N \underset{s}{\sum} \sum_{j} \mid \int_{I_{k s 0}} p_{j}(\xi) d \xi-\text { meas } \varepsilon_{k s j_{0}} \mid \\
& +\eta_{1} \underset{s}{\sum} \int_{I_{k s 0}} \sum_{j} p_{j}(\xi) d \xi+\eta_{1} \underset{s}{\sum} \sum_{j} \int_{\varepsilon_{k s j 0}} d \xi \\
& \leqq 2 N \eta_{2}+2 \eta_{1} b=\sigma+\sigma=2 \sigma .
\end{aligned}
$$

$$
|x(t)-y(t)| \leqq\left|\mu_{1}\right|+\left|\mu_{2}\right| \leqq 2 \sigma+\sigma=3 \sigma \leqq \varepsilon_{1}=\min [d, \varepsilon, \delta]
$$

and $(t, x(t)) \in A_{0}$ for all $0 \leqq t<\bar{t}$. Thus, $x(t)$ is defined in all of $[0, b]$, the graph $(t, x(t))$ lies in $A_{0}$, and

$$
\begin{array}{cc}
x(t)=x_{0}+\int_{0}^{t} f(t, y(\xi), u(\xi)) d \xi, & u(t) \in U(t, y(t)), \\
& |x(t)-y(t)| \leqq \min [d, \varepsilon, \delta], \quad 0 \leqq t \leqq b .
\end{array}
$$

By property $\left(p_{4}\right)$ there is now for every $t$ at least one point $u_{0}(t)$ such that

$$
u_{0}(t) \in U(t, x(t)), \quad\left|u_{0}(t)-u(t)\right| \leqq H|x(t)-y(t)| \leqq H \delta .
$$

By the usual Filippov's argument we can select $u_{0}(t), 0 \leqq t \leqq b$, so as to make it measurable in $[0, b]$. Also $\left|u_{0}(t)\right| \leqq|u(t)|+H \delta \leqq N+H \delta<N+1$.

(b) If we denote by $x_{0}(t)$ the function $x(t)$ determined above we have

$$
\begin{gathered}
x_{0}(t)=x_{0}+\int_{0}^{t} f(\xi, y(\xi), u(\xi)) d \xi, \quad u(t) \in U(t, y(t)), \\
\left|x_{0}(t)-y(t)\right| \leqq \varepsilon_{1}=\min [d, \varepsilon, \delta], \\
u_{0}(t) \in U\left(t, x_{0}(t)\right), \quad\left|u_{0}(t)-u(t)\right| \leqq H \delta, \quad 0 \leqq t \leqq b .
\end{gathered}
$$

Now $f\left(t, x_{0}(t), u_{0}(t)\right), 0 \leqq t \leqq b$, exists, and by integration we obtain a new function

$$
x_{1}(t)=x_{0}+\int_{0}^{t} f\left(\xi, x_{0}(\xi), u_{0}(\xi)\right) d \xi, \quad 0 \leqq t \leqq b
$$

By Lipschitz condition we have

$$
\begin{aligned}
\left|x_{1}(t)-x_{0}(t)\right| & \leqq \int_{0}^{t}\left[L\left|x_{0}(\xi)-y(\xi)\right|+L\left|u_{0}(\xi)-u(\xi)\right|\right] d \xi \\
& \leqq L \delta t+L H \delta t=L(H+1) \delta t \leqq L(H+1) \delta b
\end{aligned}
$$


Thus, $\left|x_{1}(t)-y(t)\right| \leqq \delta+L(H+1) \delta b$, hence $\left(t, x_{1}(t)\right) \in A_{0}$. We have $\left(t, x_{1}(t)\right) \in A$, $-N_{0} \leqq t \leqq N_{0},\left|x_{0}(t)\right| \leqq N_{0},\left|x_{1}(t)\right| \leqq N_{0}, \quad u_{0}(t) \in U\left(t, x_{0}(t)\right),\left|u_{0}(t)\right| \leqq N_{0}$, and hence, by property $\left(p_{4}\right)$ there is a point $u_{1}(t)$ such that

$$
\begin{gathered}
u_{1}(t) \in U\left(t, x_{1}(t)\right), \\
\left|u_{1}(t)-u_{0}(t)\right| \leqq H\left|x_{1}(t)-x_{0}(t)\right| \leqq L H(H+1) \delta t \leqq L H(H+1) \delta b,
\end{gathered}
$$

and hence

$$
\left|u_{1}(t)\right| \leqq\left|u_{0}(t)\right|+\left|u_{1}(t)-u_{0}(t)\right| \leqq N+\delta H(1+L(H+1) b)<N+1 .
$$

By Filippov's argument we can select $u_{1}(t)$ so as to make it measurable in $[0, b]$.

By repeating this process we obtain two sequences of functions $\left[x_{k}(t)\right],\left[u_{k}(t)\right]$ such that

$$
\begin{gathered}
x_{k}(t)=x_{0}+\int_{0}^{t} f\left(\xi, x_{k-1}(\xi), u_{k-1}(\xi)\right) d \xi \\
u_{k}(t) \in U\left(t, x_{k}(t)\right), \\
\left|x_{k}(t)-x_{k-1}(t)\right| \leqq L^{k}(H+1)^{k} \delta t^{k} / k !, \\
\left|u_{k}(t)-u_{k-1}(t)\right| \leqq H L^{k}(H+1)^{k} \delta t^{k} / k !, \quad 0 \leqq t \leqq b, k=1,2, \cdots,
\end{gathered}
$$

and hence

$$
\begin{aligned}
& \left|x_{k}(t)-y(t)\right| \leqq \delta\left[1+L(H+1) b+\cdots+L^{k}(H+1)^{k} b^{k} / k !\right] \\
& \left|u_{k}(t)\right| \leqq N+\delta H\left[1+L(H+1) b+\cdots+L^{k}(H+1)^{k} b^{k} / k !\right]
\end{aligned}
$$

Thus

$$
\begin{gathered}
\left|x_{k}(t)-y(t)\right| \leqq \delta e^{L(H+1) b} \leqq \min [d, \varepsilon], \\
\left|u_{k}(t)\right| \leqq N+\delta H e^{L(H+1) b} \leqq N+1, \quad 0 \leqq t \leqq b, k=1,2, \cdots,
\end{gathered}
$$

and thus all graphs $\left(t, x_{k}(t)\right)$ lie in $A_{0}, u_{k}(t) \in U\left(t, x_{k}(t)\right)$, and $\left(t, x_{k}(t), u_{k}(t)\right) \in M_{1}$. The process can be repeated indefinitely, and relations (8) show that the sequences $\left[x_{k}(t)\right],\left[u_{k}(t)\right]$ converge uniformly toward functions $x(t), u(t)$, and relations (9) yield

$$
|x(t)-y(t)| \leqq \min [d, \varepsilon], \quad|u(t)| \leqq N+1 .
$$

Also, relations $u_{k}(t) \in U\left(t, x_{k}(t)\right)$ yield $u_{k}(t) \in U(t, x(t), c)$ for every $c>0$ and $k$ sufficiently large, hence $u(t) \in U(t, x(t), c)$ and $x(t) \in \bigcap_{c} U(t, x(t), c)$. By property (U) we have $u(t) \in U(t, x(t))$. Thus $f(t, x(t), u(t))$ is defined, and $f\left(t, x_{k}(t), u_{k}(t)\right)$ $\rightarrow f(t, x(t), u(t))$ uniformly in $[0, b]$. By (8) we obtain

$$
x(t)=x_{0}+\ell \int_{0}^{t} f(\xi, x(\xi), u(\xi)) d \xi, \quad 0 i \leqq t \leqq b .
$$


Also, $x(t)$ is absolutely continuous in $[0, b], u(t) \in U(t, x(t)), d x / d t=f(t, x(t), u(t))$, and $[x(t), u(t)]$ is an element of $\Omega$ with $|x(t)-y(t)| \leqq \varepsilon$. Statement (iii) is thereby proved, in the general case for the vase $U$ depends on both $t$ and $x$.

(c) If $U$ depends on $t$ only, then we take $H=0$ in part (a), and then part (a) of the previous argument suffices, provided we determine $x(t)$ by solving the differential equation

$$
d x / d t=f(t, x(t), u(t)), \quad 0 \leqq t \leqq b,
$$

with $x(0)=x_{0}$ instead of equation (4). Here $u(t) \in U(t, y(t))=U(t)$, and hence also $u(t) \in U(t, x(t))$. The argument is the same provided we add the term

$$
\int_{0}^{t}[f(\xi, y(\xi), u(\xi))-f(\xi, x(\xi), u(\xi))] d \xi
$$

to the second member of (6), and then (7) becomes

$$
|x(t)-y(t)| \leqq 3 \sigma+\int_{0}^{t} L|x(\xi)-y(\xi)| d \xi,
$$

and by Gronwall's lemma

$$
|x(t)-y(t)| \leqq 3 \sigma e^{L b} \leqq \varepsilon_{1}=\min [d, \varepsilon, \delta]
$$

Then $x(t), u(t), t_{1} \leqq t \leqq t_{2}$, is the required pair, and statement (iii) is proved again for $U$ depending on $t$ only, with no use of property $\left(p_{4}\right)$ and no use of the first part of property $\left(p_{3}\right)$. The process so simplified reduces to the one proposed by R.V. Gamkrelidze [3] for this and other questions (chattering process).

REMARK. The approximation of a generalized trajectory $y(t)$ by means of usual trajectories $x(t)$ by the use of the "chattering" process of the part (a) of the proof of (iii) may require suitable sets $\varepsilon_{k s j}$ instead of arbitrary ones when the components $u^{(j)}(t)$ of the generalized strategy $v(t)$ are not bounded and $f$ is not uniformly Lipschitzian in $x$. This can be seen by the following simple example where the trajectories $x_{k}(t)$ obtained by the chattering process on a given subdivision of $I=[0,1]$ into equal parts does not converge uniformly toward $y(t)$.

Let $m=n=1, A=E_{2}, U=E_{1}$. Thus $U$ is a fixed set (and certainly satisfies properties $(U)$ and $\left.\left(p_{4}\right)\right)$. Let $K_{1}, K_{2}$ be the two subsets of the $t x u$-space $E_{3}$ defined by

$$
\begin{aligned}
& K_{1}=[(t, x, u) \mid u x \geqq 0,0 \leqq t \leqq 1 / 2, \text { and } u x \leqq 0,1 / 2 \leqq t \leqq 1], \\
& K_{2}=\left[(t, x, u)|u x<0,| u|\geqq 1,| u x \mid \geqq 8^{-1}, 0 \leqq t \leqq 1 / 4\right] .
\end{aligned}
$$

Both sets $K_{1}$ and $K_{2}$ are closed, locally compact, and disjoint. Let us define the scalar function $f(t, x, u)$ in $E_{3}$. We take $f(t, x, u)=u$ for $(t, x, u) \in K_{1}$, we take $f(t, x, u)=-u^{4}\left(2^{-4}+t^{2} u^{4}\right)^{-1}$ for $(t, x, u) \in K_{2}$, and $f$ can be extended by continuity everywhere in $E_{3}$. For $0<\varepsilon<2^{-3}$ and $u=-(8 \varepsilon)^{-1}$ we have $f(0,0, u)$ 
$=-(8 \varepsilon)^{-1}, f(0, \varepsilon, u)=-(4 \varepsilon)^{-4}$, and obviously $f$ is not uniformly Lipschitzian in $x$. Now take $v=2$, and

$$
u^{(1)}(t)=2^{-1}\left(t-t^{2}\right)^{-1 / 2}(1-2 t), u^{(2)}(t)=-u^{(1)}(t), \quad 0<t<1 .
$$

Then, $\left(t, x, u^{(1)}(t)\right) \in K_{1}$ for $x \geqq 0$, and $\left(t, x, u^{(2)}(t)\right) \in K_{1}$ for $x \leqq 0$, hence

$$
\begin{array}{ll}
f\left(t, x, u^{(1)}(t)\right)=u^{(1)}(t) & \text { for } 0<t<1, x \geqq 0, \\
f\left(t, x, u^{(2)}(t)\right)=u^{(2)}(t) & \text { for } 0<t<1, x \leqq 0 .
\end{array}
$$

By integration we see that

$$
x^{(1)}(t)=\left(t-t^{2}\right)^{1 / 2}, \quad x^{(2)}(t)=-\left(t-t^{2}\right)^{1 / 2}, \quad 0 \leqq t \leqq 1,
$$

are usual trajectories joining $(0,0)$ and $(1,0)$. For the corresponding generalized trajectory with $p_{1}(t)=p_{2}(t)=1 / 2$, we have $v(t)=\left(u^{(1)}(t), u^{(2)}(t)\right)$, and

$$
g(t, y(t), p(t), v(t))=0, \quad y(t) \equiv 0, \quad 0 \leqq t \leqq 1 .
$$

For any $a, 0<a<2^{-4}$, and $a \leqq t \leqq 2 a, u^{(1)}(t)$ is positive and decreasing, hence

$$
u^{(1)}(a) \geqq u^{(1)}(t) \geqq u^{(1)}(2 a),
$$

and since $0<a<2^{-4}$, we have

$$
\begin{aligned}
& u^{(1)}(a)=2^{-1}\left(a-a^{2}\right)^{-1 / 2}(1-2 a) \leqq 2^{-1} a^{-1 / 2}(1-a)^{-1 / 2}<2^{-1} a^{-1 / 2}(2)=a^{-1 / 2}, \\
& u^{(1)}(2 a)=2^{-1}\left(2 a-4 a^{2}\right)^{-1 / 2}(1-4 a)>2^{-1}(2 a)^{-1 / 2}(2)^{-1 / 2}=2^{-2} a^{-1 / 2}>1 .
\end{aligned}
$$

Let us take $u(t)=u^{(2)}(t)$ for $0<t<a$, and $u(t)=u^{(1)}(t)$ for $a<t<2 a$, and thus the intervals $(0, a),(a, 2 a)$ are two sets $\varepsilon_{k s j}$. If $x(t)$ is the corresponding trajectory with $x(0)=0$, then $x(t)=x^{(2)}(t)$ for $0 \leqq t \leqq a$. Let $x_{0}(t)$ denote the function

$$
x_{0}(t)=x(a)-(17 \mathrm{a})^{-1}+(17 t)^{-1}, \quad a \leqq t \leqq 2 a .
$$

We have

$$
x(a) \geqq x_{0}(t) \geqq x(a)-(34 a)^{-1}, \quad a \leqq t \leqq 2 a,
$$

with

$$
-x_{0}(a)=\left(a-a^{2}\right)^{1 / 2}=a^{1 / 2}(1-a)^{1 / 2}>2^{-1 / 2} a^{1 / 2},
$$

or

$$
-x_{0}(t) \geqq-x_{0}(a) \geqq 2^{-1 / 2} a^{1 / 2} \text {, }
$$

and

$$
u^{(1)}(t) \geqq 1,\left(-x_{0}(t)\right)\left(u^{(1)}(t)\right) \geqq\left(2^{-1 / 2} a^{1 / 2}\right)\left(2^{-2} a^{-1 / 2}\right)>2^{-3}
$$


Thus the graph $\left(t, x_{0}(t), u^{(1)}(t)\right)$ lies in $K_{2}$. Also, for $a \leqq t \leqq 2 a$, and $u=u^{(1)}(t)$, we have

$$
\begin{aligned}
u^{4}\left(2^{-4}+t^{2} u^{4}\right)^{-1} & =\left(t^{2}+(2 u)^{-4}\right)^{-1} \geqq\left[t^{2}+\left(2 u^{(1)}(2 a)\right)^{-4}\right]^{-1} \\
& \geqq\left(t^{2}+16 a^{2}\right)^{-1} \geqq 17^{-1} t^{-2}
\end{aligned}
$$

and hence

$$
\begin{aligned}
x(t) & =x(a)-\int_{a}^{t}\left(u^{(1)}(\xi)\right)^{4}\left(2^{-4}+\xi^{2}\left(u^{(1)}(\xi)\right)^{4}\right)^{-1} d \xi \\
& \leqq x(a)-\int_{a}^{t}(17)^{-1} \xi^{-2} d \xi=x(a)-(17 a)^{-1}+(17 t)^{-1}=x_{0}(t), \\
& a \leqq t \leqq 2 a .
\end{aligned}
$$

Thus $\left(t, x(t), u^{(1)}(t)\right) \in K_{2}$ for $t=a$, as well as for all $a \leqq t \leqq 2 a$. Now

$$
x(2 a) \leqq x(a)-(34)^{-1} a^{-1} \leqq-2^{-1 / 2} a^{1 / 2}-(34)^{-1} a^{-1},
$$

and $x(2 a) \rightarrow-\infty$ as $a \rightarrow 0+$. This proves that the simple chattering process with arbitrary subsets $\varepsilon_{k s j}$ does not always assure that $x(t) \rightarrow y(t)$ uniformly, when $v$ is unbounded and $f$ is not uniformly Lipschitzian in $x$.

(iv) Let $A$ be any closed subset of $E_{1} \times E_{n}$, let $U(t, x)$ be any closed subset of $E_{m}$ for every $(t, x) \in A$, satisfying condition $(U)$ in $A$, and let $\tilde{f}(t, x, u)$ be any continuous vector function on $M$. Assume that properties $\left(p_{1}\right),\left(p_{2}\right),\left(p_{3}\right),\left(p_{4}\right)$ hold. Then property $(P)$ holds. If $U(t, x)$ depends on $t$ only, then property $\left(p_{4}\right)$ is trivial and property $\left(p_{3}\right)$ is the usual Lipschitz condition in $x$.

Proof. This statement is an immediate consequence of properties $\left(p_{1}\right),\left(p_{2}\right)$ and (iii).

In the proof of Theorem VI we shall consider the subclass $\Omega_{0}$ of all admissible pairs $[x(t), u(t)]$ already in $\Omega$ (that is, satisfying the boundary conditions) and for which

$$
\int_{t_{1}}^{t_{2}}\left|d x^{i} / d t\right|^{p} d t \leqq N_{i}, \quad i=1, \cdots, n,
$$

for certain constants $N_{i}$. We shall also denote by $\Omega_{0}^{*}$ the analogous subclass of generalized elements $[y(t), p(t), v(t)]$ already in $\Omega^{*}$. We have $\Omega_{0} \subset \Omega_{0}^{*}$. Hence, if $i_{0}$ denotes the infimum of $I[x, u]$ in $\Omega_{0}$ and $j_{0}$ the one of $J[y, p, u]$ in $\Omega_{0}^{*}$, we have $i_{0} \geqq j_{0}$. By property $\left(P_{0}\right)$ we shall mean the equality $i_{0}=j_{0}$.

Analogously we shall denote by property $\left(p_{10}\right)$ the property analogous to $\left(p_{1}\right)$ where in addition we require

$$
\left|\int_{t_{1}}^{t_{2}}\right| d x^{i} /\left.d t\right|^{p} d t-\int_{t^{\prime} 1_{1}}^{t^{\prime} z_{2}}\left|d y^{i} / d t\right|^{p} d t \mid \leqq \varepsilon, \quad i=1, \cdots, n .
$$


Also, if we introduce the class $\Omega_{\delta 0}$ of all admissible pairs $[x(t), u(t)]$ already in $\Omega_{\delta}$ and for which

$$
\int_{t_{1}}^{t_{2}}\left|d x^{i} / d t\right|^{p} d t \leqq N_{i}+\delta, \quad i=1, \cdots, n,
$$

then a number $i_{0}^{\prime}$ can be defined by

$$
i_{0}^{\prime}=\lim _{\delta \rightarrow 0} \operatorname{Inf}_{\Omega_{\delta 0}} I[x, u] .
$$

Obviously, $i_{0} \geqq i_{0}^{\prime}$. We shall denote by property $\left(p_{20}\right)$ the equality $i_{0}=i_{0}^{\prime}$. Then the statements (iii)* and (iv)* below are the analogues of statements (iii) and (iv).

(iii)* Under the hypotheses of (iii) with $\Omega^{*}, \Omega$ replaced by $\Omega_{0}^{*}, \Omega_{0}$, we can choose $\left[\tilde{x}(t)=\left(x^{0}, x\right), u(t), t_{1} \leqq t \leqq t_{2}\right]$ in such a way that, in addition, we have also

$$
\int_{t_{1}}^{t_{2}}|| d x^{i} /\left.d t\right|^{p}-\left|d y^{i} / d t\right|^{p} \mid d t \leqq \varepsilon, \quad i=1, \cdots, n .
$$

(iv)* Under the hypotheses of (iv) with $\Omega^{*}, \Omega$ replaced by $\Omega_{0}^{*}, \Omega_{0}$, and with $\left(p_{1}\right)$ and $\left(p_{2}\right)$ replaced by $\left(p_{10}\right)$ and $\left(p_{20}\right)$, then property $\left(P_{0}\right)$ holds.

The proofs are modifications of the ones for statements (iii) and (iv).

\section{Existence theorems for weak solutions.}

EXISTENCE THEOREM V (FOR WEAK SOLUTION). Let $A$ be a compact subset of the tx-space $E_{1} \times E_{n}$, let $U(t, x)$ be a closed subset of the $U$-space $E_{m}$ for every $(t, x) \in A$, and let $\tilde{f}(t, x, u)=\left(f_{0}, f\right)=\left(f_{0}, f_{1}, \cdots, f_{n}\right)$ be a continuous vector function on the set $M$ of all $(t, x, u)$ with $(t, x) \in A, u \in U(t, x)$. Let us assume that there is some continuous scalar function $\Phi(\zeta), 0 \leqq \zeta<+\infty$, with $\Phi(\zeta) / \zeta \rightarrow+\infty$ as $\zeta \rightarrow+\infty$, such that $f_{0}(t, x, u) \geqq \Phi(|u|)$ for all $(t, x, u) \in M$ and that there are constants $C, D \geqq 0$ such that $|f(t, x, u)| \leqq C+D|u|$ for all $(t, x, u) \in M$. Let us assume that $U(t, x)$ satisfies property $(U)$ in $A$, that $\widetilde{R}(t, x)$ satisfies property $(Q)$ in $A$, and that property $(P)$ holds. Let $\Omega$ be the class of all admissible pairs $x(t), u(t), t_{1} \leqq t \leqq t_{2}$, satisfying given boundary conditions $\left(t_{1}, x\left(t_{1}\right), t_{2}, x\left(t_{2}\right)\right) \in B$, where $B$ is a closed subset of the $t_{1} x_{1} t_{2} x_{2}$-space $E_{2 n+2}$. Let us assume that $\Omega$ is not empty.Then the infimum $i$ of $I[x, u]$ in $\Omega$ is attained by a weak solution [that is, $i$ is attained by $J[x, p, v]$ in the class $\left.\Omega^{*}\right]$.

When $A$ is not compact, but closed, then the theorem still holds under the additional hypotheses stated at the end of Existence Theorem I.

Proof. The proof proceeds exactly as the one of Theorem I of $\S 7$. First we prove that the infimum $i$ of $I[x, u]$ in $\Omega$ is finite, then we take a sequence of pairs $x_{k}(t), u_{k}(t), t_{1 k} \leqq t \leqq t_{2 k}, k=1,2, \cdots$, of the class $\Omega$ such that

$$
i \leqq I\left[x_{k}, u_{k}\right] \leqq i+k^{-1} \leqq i+1, \quad k=1,2, \cdots,
$$


and we introduce the auxiliary variable $x_{k}^{0}(t)$ with $x_{k}^{0}\left(t_{1 k}\right)=0, d x_{k}^{0} / d t=f_{0}\left(t, x_{k}(t), u_{k}\right)$ a.e. in $\left[t_{1 k}, t_{2 k}\right]$, so that $I\left[x_{k}, u_{k}\right]=x_{k}^{0}\left(t_{2 k}\right) \rightarrow i$. Then, we show as in the proof of Theorem I that there exists a subsequence, say still $\left[x_{k}^{0}(t), x_{k}(t)\right]$ which converges in the mode indicated there toward $\left[Y(t)+Z(t), x(t), t_{1} \leqq t \leqq t_{2}\right], Y(t), x(t)$ absolutely continuous and $Z(t)$ singular in $\left[t_{1}, t_{2}\right]$, with $Y\left(t_{2}\right) \leqq i, Y\left(t_{1}\right)=0$.

We shall now interpret the elements $x_{k}^{0}(t), x_{k}(t), u_{k}(t)$ with $\left[x_{k}(t), u_{k}(t)\right]$ in the class $\Omega$, as corresponding to systems $p_{k}(t)=\left(p_{j k}, j=1, \cdots, v\right), x_{k}(t), v_{k}(t)=$ $\left(u_{k}^{(j)}(t), j=1, \cdots, v\right)$ of the class $\Omega^{*}$ with $p_{j k}(t)=1 / v, u_{k}^{(j)}(t)=u_{k}(t), j=1, \cdots, v$, for every $k$. Thus, if $\tilde{g}=\left(g_{0}, g\right), \tilde{x}=\left(x^{0}, x\right)$, then

$$
\begin{aligned}
& d \tilde{x}_{k} / d t=\tilde{g}\left(t, x_{k}(t), p_{k}(t), v_{k}(t)\right), \quad \text { a.e. in }\left[t_{1 k}, t_{2 k}\right], \\
& p_{k}(t) \in \Gamma, \quad v_{k}(t) \in V\left(t, x_{k}(t)\right), \quad\left(t, x_{k}(t)\right) \in A .
\end{aligned}
$$

We shall now apply Closure Theorem II to the differential system $d \tilde{x} / d t=\tilde{g}$ and relative constraints. Since the set $\tilde{R}(t, x)$ is convex for every $(t, x)$ by force of (ii) of $\S 15$ and satisfies condition $(Q)$ in $A$ by hypothesis, we conclude that there exists a pair of measurable functions $p(t), v(t), t_{1} \leqq t \leqq t_{2}$, such that

$$
\begin{array}{cc}
d Y / d t=g_{0}(t, x(t), p(t), v(t)), & \\
d x / d t=g(t, x(t), p(t), v(t)), & \text { a.e. in }\left[t_{1}, t_{2}\right], \\
p(t) \in \Gamma, v(t) \in V(t, x(t)), & (t, x(t)) \in A .
\end{array}
$$

Hence,

$$
J[x, p, v]=\int_{t_{1}}^{t_{2}} g_{0}(t, x(t), p(t), v(t)) d t=Y\left(t_{2}\right) \leqq i .
$$

On the other hand, $J[x, p, v] \geqq j$ and $j=i$ by property $(P)$. Hence, $J=i$ and Theorem $\mathrm{V}$ is thereby proved.

EXISTENCE THEOREM VI (FOR WEAK SOlutions). Let $A$ be a compact subset of the tx-space $E_{1} \times E_{n}$, and for every $(t, x) \in A$ let $U(t, x)$ be a closed subset of the $u$-space $E_{m}$. Let $f(t, x, u)=\left(f_{0}, f_{1}, \cdots, f_{n}\right)$ be a continuous vector function in the set $M$ of all $(t, x, u)$ with $(t, x) \in A, u \in U(t, x)$. Let us assume that the set $U(t, x)$ satisfies property $(U)$ in $A$, and that the set $\tilde{R}(t, x)$ satisfies property $(Q)$ in $A$. Let us assume that $f_{0}(t, x, u) \geqq-G$, for some constant $G \geqq 0$ and all $(t, x, u) \in M$. Let $\Omega_{0}$ be the class of all admissible pairs $\left[\tilde{x}(t)=\left(x^{0}, x\right), u(t)\right]$ of $\Omega$ satisfying given boundary conditions $\left(t_{1}, x\left(t_{1}\right), t_{2}, x\left(t_{2}\right)\right) \in B$, where $B$ is a closed subset of the $t_{1} x_{1} t_{2} x_{2}$-space $E_{2 n+2}$, and satisfying the inequalities

$$
\int_{t_{1}}^{t_{2}}\left|d x^{i} / d t\right|^{p} d t \leqq N_{i}, \quad i=1,2, \cdots, n,
$$

for some constants $N_{i} \geqq 0, p>1$. Let $\Omega_{0}^{*}$ be the analogous subclass of all the generalized elements $\left[\tilde{y}(t)=\left(y^{0}, y\right), p(t), v(t)\right]$ of $\Omega^{*}$ satisfying the same 
boundary conditions and inequalities. Assume that $\Omega_{0}$ is not empty and property $\left(P_{0}\right)$ holds. Then the infimum $i$ of $I[x, u]$ in $\Omega_{0}$ is attained by a weak solution [that is, $i$ is attained by $J[y, p, v]$ in $\left.\Omega_{0}^{*}\right]$.

When $A$ is not compact, but $A$ is closed, then Theorem VI still holds under the additional hypotheses stated at the end of Theorem II.

As for Theorem II, every inequality (1), which is a consequence of a relation of the form $I \leqq N$, can be disregarded.

REMARK. For free problems, that is, $m=n, U=E_{n}, f=u$, property $\left(p_{4}\right)$ is trivial and of property $\left(p_{3}\right)$ we need only verify the second part for the scalar function $f_{0}$. If in addition $A=E_{1} \times E_{n}$, then property $\left(p_{1}\right)$ is trivial. Statements $\mathrm{V}$ and VI (and corresponding additional hypotheses for the case $A$ not compact but closed) yield analogous statements for free problems.

For Pontryagin's problems, that is, $U(t, x)$ compact for every $(t, x) \in A$ and an upper semicontinuous function of $(t, x)$ in $A$, then $\widetilde{R}(t, x)$ is certainly compact for every $(t, x) \in A$ and an upper semicontinuous function of $(t, x)$ in $A$, and hence satisfies property $(Q)$. Growth conditions on $f_{0}$ and $f$ also are trivially satisfied. Statements V and VI (and corresponding hypotheses for the case $A$ not compact but bounded) yield analogous statements for Pontryagin's problems.

\section{REFERENCES}

1. L. Cesari, (a) Semicontinuitá e convessitá nel calcolo delle variazioni, Ann. Scuola Norm. Sup. Pisa (3) 18 (1964), 389-423.

(b) Un teorema di esistenza in problemi di controlli ottimi, ibid. (3) 19 (1965), 35-78.

(c) An existence theorem in problems of optimal control, J. Soc. Indust. Appl. Math. Ser. A Control 3 (1965), 7-22.

(d) Existence theorems for optimal solutions in Pontryagin and Lagrange problems, J. Soc. Indust. Appl. Math. Ser. A Control 3 (1965), 475498.

(e) Existence theorems for weak and usual optimal solutions in Lagrange problems with unilateral constraints. I, Trans. Amer. Math. Soc. 124 (1966), 369-412.

2. A. F. Filippov, On certain questions in the theory of optimal control, Vestnik Moskov. Univ. Ser. Mat. Meh. Astronom. 2 (1959), 25-32 = J. Soc. Indust. Appl. Math. Ser. A Control 1 (1962), 76-84.

3. R. V. Gamkrelidze, On sliding optimal states, Dokl. Akad. Nauk SSSR 143 (1962). 1243-1245=Soviet Math. Dokl. 3 (1962), 559-561.

4. L. Markus and E. B. Lee, Optimal control for nonlinear processes, Arch. Rational Mech, Anal. 8 (1961), 36-58.

5. E. J. McShane, (a) Curve-space topologies associated with variational problems, Ann. Scuola Norm. Sup. Pisa (2) 9 (1940), 45-60.

(b) Generalized curves, Duke Math. J. 6 (1940), 513-536.

(c) Necessary conditions in the generalized-curve problem of the calculus of variations, ibid. 7 (1940), 1-27.

(d) Existence theorems for Bolza problems in the calculus of variations, ibid. 28-61.

(e) A metric in the space of generalized curves, Ann. of Math. 52 (1950), 328-349.

6. M. Nagumo, Uber die gleichmässige Summierbarkeit und ihre Anwendung auf ein Variationtions-problem, Japan. J. Math. 6 (1929), 173-182. 
7. L. S. Pontryagin, Optimal control processes, Uspehi Mat. Nauk 14 (1959), 3-20; Amer. Math. Soc. Transl. (2) 18 (1961), 321-339.

8. L. S. Pontryagin, V.C. Boltyanskii, R. V. Gamkrelidze and E. F. Mishchenko, The mathematical theory of optimal processes, Gosudarst, Moscow, 1961 ; English transl., Interscience, New York, 1962; Pergamon, New York, 1964.

9. E. O. Roxin, The existence of optimal controls, Michigan Math. J. 9 (1962), 109-119.

10. L. Tonelli, (a) Sugli integrali del calcolo delle variazioni in forma ordinaria, Ann. Scuola Norm. Sup. Pisa (2) 3 (1934), 401-450; Opere Scelte, Vol. 3, Edizioni Cremonese, Rome, 1962, 192-254;

(b) Un teorema di calcolo delle variazioni, Rend. Accad. Naz. Lincei 15 (1932), 417-423; Opere Scelte, Vol. 3, ibid., 84-91.

(e) Fondamenti di calcolo delle variazioni, Zanichelli, Bologna, 1921-1923, 2 volumes.

11. L. Turner, The direct method in the calculus of variations, Ph. D. Thesis, Purdue Univ., Lafayette, Indiana, 1957.

12. J. Warga, Relaxed variational problems, J. Math. Anal. Appl. 4 (1962), 111-128.

13. T. Wazewski, (a) Sur une généralization de la notion des solutions d'une équation au contingent, Bull. Acad. Polon. Sci. Sér. Math. Astronom. Phys. 10 (1962), 11-15.

(b) Sur les systèmes de commande non linéaires dont le contredomaine de commande n'est pas forcément convexe, ibid. 10 (1962), 17-21.

14. L. C. Young, Generalized curves and the calculus of variations, C. R. Soc. Sci. Varsovie (3) 30 (1937), 211-234.

\section{UNIVERSITY OF MICHIGAN,}

ANN ARbor, Michigan 